\title{
Effects of Tridocosahexaenoyl-Glycerol Emulsion on Proteinuria in Rats with Nephrotoxic Serum Nephritis
}

\author{
Norio Nakamura ${ }^{a}$ b Ryuichiro Kumasaka ${ }^{b}$ Lu Yong Fu $^{\mathrm{b}}$ \\ Takeshi Fujita $^{\mathrm{b}}$ Reiichi Murakami $^{\mathrm{b}}$ Michiko Shimada $^{\mathrm{b}}$ \\ Yuko Shimaya $^{b}$ Hiroshi Osawa $^{\mathrm{b}}$ Hideaki Yamabe $^{\mathrm{b}}$ \\ Ken Okumura $^{\mathrm{b}}$ Kei Hamazaki ${ }^{\mathrm{c}}$ Tomohito Hamazaki ${ }^{\mathrm{d}}$
}

${ }^{a}$ Community Medicine and ${ }^{b}$ Department of Nephrology, Hirosaki University Graduate School of Medicine, Hirosaki, and ' Department of Public Health, Faculty of Medicine, and dDepartment of Clinical Sciences, Institute of Natural Medicine, University of Toyama,

Toyama, Japan

\section{Key Words}

Docosahexaenoic acid • Eicosapentaenoic acid • Emulsion • Nephrotoxic serum nephritis •

Polyunsaturated fatty acids

\begin{abstract}
Background: Docosahexaenoic acid (DHA) is one of the $n-3$ polyunsaturated fatty acids and an important component of cell membrane phospholipids (PL). Nephrotoxic serum (NTS) nephritis was a worldwide model of the Goodpasture syndrome. We investigated the effects of tridocosahexaenoyl-glycerol (DHA-TG) emulsion on proteinuria in rats with NTS nephritis. Methods: Sixteen male Wistar rats weighing approximately $200 \mathrm{~g}$ were used. Twelve rats were treated with NTS via the tail vein and divided into 3 groups (groups A, B, and C). Another 4 rats treated with saline served as controls (group D). DHA-TG and soybean oil emulsions were intraperitoneally administered to the rats in groups A and B, respectively, $24 \mathrm{~h}$ prior to NTS injection, and 0 , $1,2,3,4$, and 5 days after the injection. Saline was administered to the rats in groups $C$ and $D$ in the same manner. All rats were sacrificed on day 6 to obtain plasma and kidney samples. Analyses of urinary protein levels and fatty acid composition of plasma and kidney as well as histological examination of the kidneys were performed. Results: Urinary protein levels in group A were significantly lower than those in group C $(35.0 \pm 13.3$ vs. $79.2 \pm 11.8 \mathrm{mg} /$ day on day 5 ,
\end{abstract}


means $\pm S E, p<0.05)$. DHA levels in the PL fraction of the kidneys in group A were significantly increased compared with those in groups $B$ and $C$. Conclusions: These results suggest that the DHA-TG emulsion may have beneficial effects on NTS nephritis in the rat.

Copyright $\odot 2011$ S. Karger AG, Basel

\section{Introduction}

Docosahexaenoic acid (DHA) is one of the $\mathrm{n}-3$ polyunsaturated fatty acids. It has 22 carbon atoms and 6 double bonds, and is an important component of cell membrane phospholipids (PL) [1]. DHA is found in the brain, nervous system, heart, and sperm of land animals, and metabolizes to some chemical mediators. In addition, it modifies the production of prostaglandins and leukotrienes [2]. Consequently, DHA might have anti-inflammatory and antiatherosclerotic effects.

Nephrotoxic serum (NTS) nephritis is a worldwide model of the Goodpasture syndrome. This model was first reported by Masugi [3] in 1933. NTS nephritis includes heterologous and autologous phases. The heterologous phase consists of activation of complements, glomerular endothelial injury, platelet aggregation, and migration of polymorphonuclear leukocytes (PMNL) [3]. The autologous phase consists of formation of an immune complex, progression of glomerular injury, proliferation of endothelial and mesangial cells, and formation of crescents [3]. We have previously reported that DHA administration decreased leukotriene $\mathrm{B}_{4}$ production by PMNL in rabbits [4]. Consequently, we hypothesized that DHA administration might suppress proteinuria in rats with NTS nephritis by preventing a reaction in the heterologous phase. In the present study, we injected tridocosahexaenoyl-glycerol (DHA-TG) emulsion into rats with NTS nephritis and investigated their urinary protein levels and fatty acid composition of plasma and kidneys.

\section{Methods}

\section{Experimental Animals}

Male Wistar rats weighing approximately $200 \mathrm{~g}$ were purchased from Clea Japan (Tokyo, Japan), maintained in our animal facility, and allowed free access to standard rat chow and tap water throughout the experiment. This study was conducted in accordance with the Guidelines for Animal Experiments of the Hirosaki University.

\section{Preparation of NTS}

Rat glomerular basement membranes obtained from perfused renal cortices were digested with trypsin (Sigma, St. Louis, Mo., USA) for $3 \mathrm{~h}$ at $37^{\circ} \mathrm{C}$. After heating the digest at $60^{\circ} \mathrm{C}$ for $30 \mathrm{~min}$, the mixture was centrifuged at 76,000 $\mathrm{g}$ for $1 \mathrm{~h}$ and the supernatant was lyophilized. To produce NTS, 2 white rabbits were subcutaneously injected with the lyophilized sample emulsified in complete Freund's adjuvant. Subsequent injections were administered 2, 4, and 6 weeks after the initial immunization. The rabbits were bled in the 7th week and sera were collected. Sera exhibiting nephrotoxic potency (demonstrated by proteinuria induction in rats after the intravenous injection of $0.2 \mathrm{ml}$ antiserum) were pooled. The pooled antiserum was heat inactivated at $56^{\circ} \mathrm{C}$ for $30 \mathrm{~min}$ and stored at $-20^{\circ} \mathrm{C}$ until use.

\section{Experimental Design}

Twelve rats were treated with NTS via the tail vein and divided into 3 groups (groups A, B, and C). Another 4 rats treated with saline served as controls (group D). DHA-TG and 
Fig. 1. Experimental design. The circles indicate administration of the same drug or reagent as used on day 0 .
Nakamura et al.: DHA-TG and NTS Nephritis

\begin{tabular}{|c|c|c|c|c|c|c|c|c|}
\hline & & $\begin{array}{c}\text { e collectior } \\
\text { Day } 0\end{array}$ & Day 1 & $\begin{array}{l}1 \\
\text { Day } 2\end{array}$ & Day 3 & Day 4 & Day 5 & Day 6 \\
\hline $\begin{array}{c}\text { Group A } \\
(\mathrm{n}=4)\end{array}$ & $\begin{array}{l}\text { i.p. } \\
\text { i.v. }\end{array}$ & $\begin{array}{c}\text { DHA } \\
\text { emulsion }\end{array}$ & $\begin{array}{c}\circ \\
\text { NTS }\end{array}$ & $\circ$ & 0 & $\circ$ & $\circ$ & \\
\hline $\begin{array}{l}\text { Group B } \\
(n=4)\end{array}$ & $\begin{array}{l}\text { i.p. } \\
\text { i.v. }\end{array}$ & $\begin{array}{c}\text { Soybean oil } \\
\text { emulsion }\end{array}$ & $\begin{array}{l}\text { il } 0 \\
\text { NTS }\end{array}$ & $\circ$ & 0 & 0 & 0 & $\begin{array}{c}\text { Sacrificed } \\
\mid\end{array}$ \\
\hline $\begin{array}{l}\text { Group C } \\
(n=4)\end{array}$ & $\begin{array}{l}\text { i.p. } \\
\text { i.v. }\end{array}$ & Saline & $\begin{array}{c}\circ \\
\text { NTS }\end{array}$ & o & 0 & o & 0 & $\begin{array}{l}\downarrow \\
\text { Kidney } \\
\text { plasma }\end{array}$ \\
\hline $\begin{array}{l}\text { Group D } \\
(n=4)\end{array}$ & $\begin{array}{l}\text { i.p. } \\
\text { i.v. }\end{array}$ & Saline & $\begin{array}{c}\circ \\
\text { Saline }\end{array}$ & 0 & o & ○ & ० & \\
\hline
\end{tabular}

soybean oil emulsions $(10 \mathrm{ml} / \mathrm{kg})$ were intraperitoneally administered to the rats in groups $\mathrm{A}$ and $\mathrm{B}$, respectively, $24 \mathrm{~h}$ prior to NTS injection and $0,1,2,3,4$, and 5 days after the injection. Saline $(10 \mathrm{ml} / \mathrm{kg})$ was administered to the rats in groups $\mathrm{C}$ and $\mathrm{D}$ in the same manner. Urine samples were obtained individually during the experiment. All rats were sacrificed on day 6 to obtain plasma and kidney samples. Urinary protein levels and fatty acid composition of plasma and kidneys were assessed, and the kidneys were examined histologically (fig. 1).

\section{Preparation of DHA-TG Emulsion}

DHA-TG (66\% pure) was donated from Kentech (Toyama, Japan). DHA-TG was fortified with $0.2 \% \alpha$-tocopherol and emulsified with egg yolk lecithin [5]. One hundred milliliters of DHA-TG emulsion contained $10 \mathrm{~g}$ DHA-TG, $1.2 \mathrm{~g}$ egg yolk lecithin as an emulsifier, and 2.5\% glycerol for osmotic pressure. The fatty acid composition of the DHA-TG emulsion was as follows: $6.5 \%$ palmitic acid (16:0), 3.4\% stearic acid (18:0), 5.5\% oleic acid (18:1n-9), 1.6\% linolenic acid (LA; 18:2n-6), 0.2\% $\alpha$-LA (18:3n-3), 2.7\% arachidonic acid (AA; 20:4n-6), 6.5\% eicosapentaenoic acid (EPA; 20:5n-3), and 65.7\% DHA (22:6n-3). The fatty acid composition of the soybean oil emulsion was as follows: $13.6 \%$ palmitic acid (16:0), 5.0\% stearic acid (18: $0), 19.3 \%$ oleic acid (18:1n-9), 50.1\% LA (18:2n-6), 9.8\% $\alpha$-LA (18:3n-3), 0.4\% AA (20:4n-6), $0.0 \%$ EPA (20:5n-3), and 0.5\% DHA (22:6n-3).

\section{Biochemical Analysis of Urine and Plasma}

Urinary protein levels as well as total protein, creatinine, and total cholesterol levels in plasma were all analyzed enzymatically.

\section{Histological Examination}

Right kidneys were obtained for light- and immunofluorescence-microscopic examinations. Each specimen was fixed in $10 \%$ buffered formalin and embedded in paraffin for lightmicroscopic examination. Sections $(2-\mu \mathrm{m}$ thick) were stained with periodic acid-Schiff. Frozen sections (5- $\mu \mathrm{m}$ thick) were cut using a cryostat and stained with fluorescein isothiocyanate (FITC)-conjugated anti-rat IgG, IgA, IgM (Bethyl Laboratories, Montgomery, Tex., USA), FITC-conjugated anti-rat $\mathrm{C}_{3}$ (MP Biomedicals, Aurora, Ohio, USA), and FITC-conjugated anti-mouse IgG (Wako, Osaka, Japan). 
Fig. 2. Urinary protein levels in the 4 groups. Twelve rats were treated with NTS via the tail vein and divided into 3 groups (groups A, B, and C). Another 4 rats treated with saline served as controls (group D). DHA-TG $(10 \mathrm{ml} / \mathrm{kg}$ ) and soybean oil $(10 \mathrm{ml} / \mathrm{kg})$ emulsions were intraperitoneally administered to the rats in groups $\mathrm{A}$ and $\mathrm{B}$, respectively, $24 \mathrm{~h}$ prior to NTS injection and $0,1,2,3,4$, and 5 days after the injection. The same dose of saline was administered to the rats in groups $\mathrm{C}$ and $\mathrm{D}$ in the same manner. Urine samples were obtained individually during the experiment (days 1 , $2,4$, and 5$)$. Means $\pm \mathrm{SE},{ }^{*} \mathrm{p}<0.01$ vs. group $\mathrm{D}$.

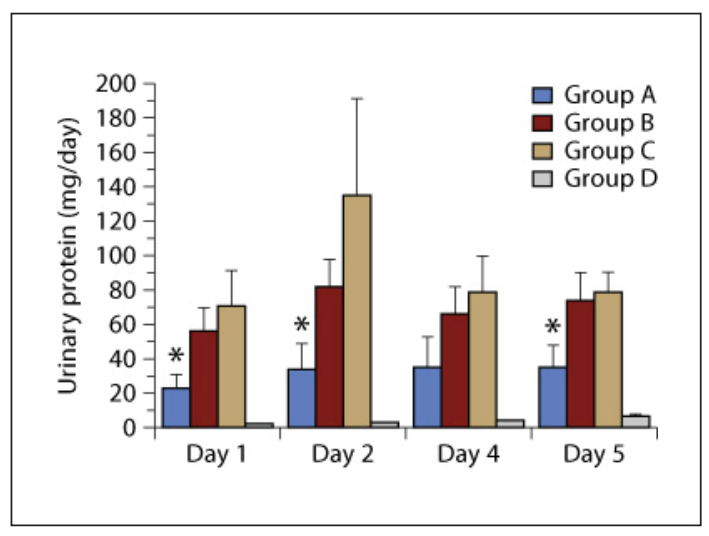

Fatty Acid Analysis of Plasma and Kidney

Left kidneys were obtained for analyzing fatty acid composition. Total lipids in a plasma or kidney homogenate in PBS were extracted according to the method of Bligh and Dyer [6]; PL, triglyceride (TG), and free fatty acid (FFA) were separated by thin-layer chromatography; after transmethylation with $\mathrm{HCl}$-methanol, the fatty acid composition was analyzed by gas chromatography (GC-14A; Shimadzu, Kyoto, Japan) with a capillary column (length, 30 m; internal diameter, $0.25 \mathrm{~mm}$; film $0.25 \mu \mathrm{m}$; J\&M Scientific, Folsom, Calif., USA); column temperature was kept at $170^{\circ} \mathrm{C}$ for the 1 st min, increased to $220^{\circ} \mathrm{C}$ at a rate of $4{ }^{\circ} \mathrm{C} / \mathrm{min}$, and kept at this temperature for $22 \mathrm{~min}$; the entire system was controlled with gas-chromatographic software (CLASS-GC10 1.3; Shimadzu).

\section{Statistical Analysis}

Data were expressed as means \pm SD or SE (fig. 1). Statistical analysis was performed by Scheffe's method. $\mathrm{p}<0.05$ was considered significant.

\section{Results}

Changes in urinary protein levels in the 4 groups are shown in figure 2. Urinary protein levels in group A were significantly lower than those in group C on days 1,2, and 5 after the injection of emulsion $(22.5 \pm 8.7$ vs. $70.7 \pm 21.5 \mathrm{mg} /$ day on day $1,34.0 \pm 14.9$ vs. $135.2 \pm$ $56.1 \mathrm{mg} /$ day on day $2,35.0 \pm 13.3 \mathrm{vs} .79 .2 \pm 11.8 \mathrm{mg} /$ day on day 5 , means $\pm \mathrm{SE}, \mathrm{p}<0.05)$.

Changes in the fatty acid composition of the PL fraction of the kidneys in the 4 groups are shown in table 1 . In group A, AA levels were significantly lower than those in groups B, $\mathrm{C}$, and D, LA levels were significantly higher than those in group C, and EPA and DHA levels were significantly higher than those in groups $B, C$, and D.

Changes in the fatty acid composition of the plasma PL fraction in the 4 groups are shown in table 1 . AA levels in group A were significantly lower than those in groups B and D. EPA levels in group A were significantly higher than those in groups B, C, and D. DHA levels in the plasma PL fraction were not significantly different among the 4 groups. Changes in the fatty acid composition of the plasma TG fraction in the 4 groups are shown in table 2. LA levels in group A were significantly lower than those in group C. EPA and DHA levels in group A were significantly higher than those in groups $\mathrm{B}, \mathrm{C}$, and $\mathrm{D}$. Changes in the fatty acid composition of the plasma FFA fraction in the 4 groups are shown in table 3 . In group A, oleic acid and LA levels were significantly lower than those in group C, AA levels 
Table 1. Changes in the fatty acid composition (mol\%) of the PL fraction in the kidney and in plasma in the 4 groups

\begin{tabular}{lcccc}
\hline \multicolumn{2}{c}{ NTS nephritis } & \multicolumn{2}{c}{ Group D } \\
\cline { 2 - 4 } & group A & group B & group C & \\
\hline Changes in the kidney & & & \\
Oleic acid & $6.95 \pm 0.16$ & $7.22 \pm 0.44$ & $6.51 \pm 0.61$ & $7.18 \pm 0.33$ \\
LA & $15.27 \pm 0.22$ & $14.68 \pm 0.61$ & $14.19 \pm 0.71^{*}$ & $15.02 \pm 0.22$ \\
AA & $23.86 \pm 0.59$ & $25.42 \pm 0.58^{*}$ & $26.62 \pm 0.18^{* *}$ & $26.15 \pm 1.00^{* *}$ \\
EPA & $1.55 \pm 0.08$ & $0.78 \pm 0.07^{* *}$ & $0.82 \pm 0.13^{* *}$ & $0.82 \pm 0.04^{* *}$ \\
DHA & $3.27 \pm 0.02$ & $2.59 \pm 0.28^{* *}$ & $2.67 \pm 0.20^{* *}$ & $2.47 \pm 0.04^{* *}$ \\
\hline Changes in plasma & & & & \\
Oleic acid & $4.05 \pm 0.21$ & $3.72 \pm 0.30$ & $3.81 \pm 0.13$ & $3.71 \pm 0.11$ \\
LA & $23.30 \pm 0.74$ & $22.51 \pm 0.83$ & $23.04 \pm 1.29$ & $24.29 \pm 0.46$ \\
AA & $13.24 \pm 0.75$ & $15.78 \pm 1.13^{* *}$ & $13.25 \pm 0.33$ & $15.15 \pm 0.67^{*}$ \\
EPA & $0.95 \pm 0.14$ & $0.53 \pm 0.05^{* *}$ & $0.38 \pm 0.05^{* *}$ & $0.52 \pm 0.04^{* *}$ \\
DHA & $4.85 \pm 0.23$ & $4.78 \pm 0.39$ & $5.36 \pm 0.98$ & $4.09 \pm 0.34$ \\
\hline
\end{tabular}

DHA emulsion (10 ml/kg; group A), $10 \mathrm{ml} / \mathrm{kg}$ of soybean oil emulsion (group B), and $10 \mathrm{ml} / \mathrm{kg}$ of saline (group C) were intraperitoneally administered to rats $24 \mathrm{~h}$ prior to NTS injection, and 0, 1, 2, 3, 4, and 5 days after the injection. Group D was the control group (no nephritis). All rats were sacrificed on day 6 to obtain plasma and kidney samples. Means $\pm \mathrm{SD}$. ${ }^{*} \mathrm{p}<0.05,{ }^{* *} \mathrm{p}<0.01$, vs. group A.

Table 2. Changes in the fatty acid composition (mol\%) of the TG fraction in plasma in the 4 groups

\begin{tabular}{lcccc}
\hline & \multicolumn{2}{l}{} & \multirow{2}{*}{ Group D } \\
\cline { 2 - 3 } & group A & group B & group C & \\
\hline Oleic acid & $19.16 \pm 0.55$ & $20.27 \pm 1.77$ & $20.62 \pm 1.40$ & $20.51 \pm 0.60$ \\
LA & $32.71 \pm 2.01$ & $34.50 \pm 2.46$ & $36.96 \pm 1.60^{*}$ & $35.82 \pm 0.40$ \\
AA & $1.71 \pm 0.30$ & $2.13 \pm 0.36$ & $2.06 \pm 0.01$ & $1.87 \pm 0.35$ \\
EPA & $2.88 \pm 0.75$ & $1.64 \pm 0.31^{*}$ & $1.57 \pm 0.30^{*}$ & $1.76 \pm 0.54^{*}$ \\
DHA & $10.58 \pm 1.45$ & $6.15 \pm 0.83^{* *}$ & $5.20 \pm 0.89^{* *}$ & $6.47 \pm 0.19^{* *}$ \\
\hline
\end{tabular}

For further information, see legend to table 1.

were significantly lower than those in groups B and C, and EPA levels were significantly higher than those in groups B, C, and D. DHA levels in the plasma FFA fraction were not significantly different among the 4 groups.

Light microscopy showed that mesangial proliferative glomerulonephritis was induced by NTS administration in groups A, B, and C, but not in group D. No significant differences were observed in histological changes in the kidneys among groups $\mathrm{A}, \mathrm{B}$, and $\mathrm{C}$ (fig. 3 ). In the immunofluorescence microscopic study, no difference was observed in the staining intensity of bound rat C3, rat IgG and mouse IgG in the glomeruli among groups A, B, and C. No staining was observed in the glomeruli of group D.

Changes in total protein, total cholesterol, and creatinine levels in the plasma in the 4 groups are shown in table 4 . Total plasma cholesterol levels in group A were significantly lower than those in groups B and C. Total protein and creatinine levels in the plasma were not significantly different among the 4 groups. 


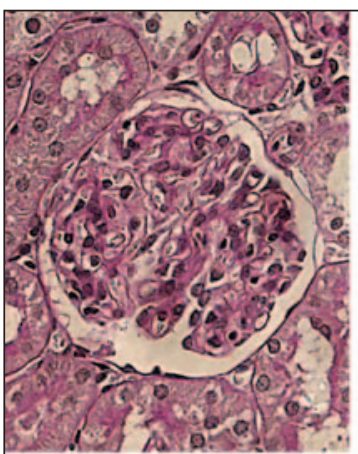

Group A

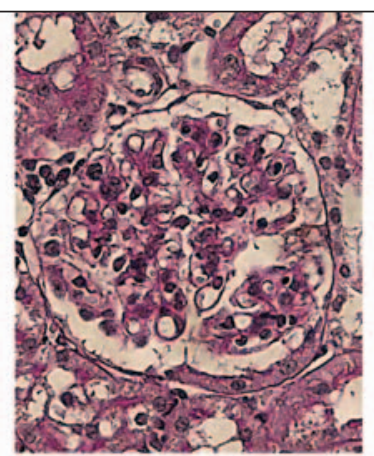

Group B

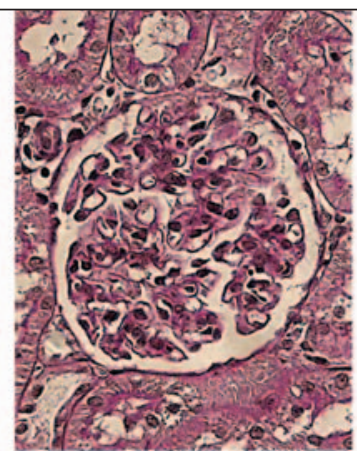

Group C

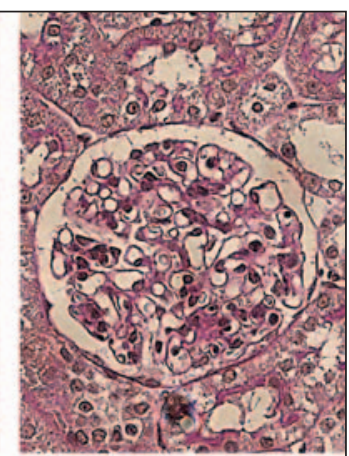

Group D

NTS nephritis

Fig. 3. Representative light-microscopic findings on day 6 in each group. Mesangial proliferative glomerulonephritis was observed in groups A, B and C. Original magnification $\times 400$.

Table 3. Changes in the fatty acid composition (mol\%) of the FFA fraction in plasma in the 4 groups

\begin{tabular}{|c|c|c|c|c|}
\hline & \multicolumn{3}{|c|}{ NTS nephritis } & \multirow[t]{2}{*}{ Group D } \\
\hline & group A & group B & group C & \\
\hline Oleic acid & $17.13 \pm 1.75$ & $16.07 \pm 1.18$ & $20.07 \pm 0.96^{*}$ & $17.97 \pm 0.79$ \\
\hline LA & $28.80 \pm 1.19$ & $27.41 \pm 2.23$ & $33.15 \pm 0.28^{* *}$ & $29.55 \pm 0.55$ \\
\hline AA & $3.43 \pm 0.44$ & $5.29 \pm 0.75^{* *}$ & $4.76 \pm 0.37^{*}$ & $4.04 \pm 0.72$ \\
\hline EPA & $1.05 \pm 0.25$ & $0.74 \pm 0.10^{*}$ & $0.69 \pm 0.11^{*}$ & $0.68 \pm 0.07^{*}$ \\
\hline DHA & $4.11 \pm 1.39$ & $3.27 \pm 0.41$ & $4.07 \pm 0.25$ & $2.86 \pm 0.25$ \\
\hline
\end{tabular}

DHA emulsion (10 ml/kg; group A), $10 \mathrm{ml} / \mathrm{kg}$ of soybean oil emulsion (group B), and $10 \mathrm{ml} / \mathrm{kg}$ of saline (group C) were intraperitoneally administered to rats $24 \mathrm{~h}$ prior to NTS injection, and 0, 1, 2, 3, 4, and 5 days after the injection. Group D was the control group (no nephritis). All rats were sacrificed on day 6 to obtain plasma and kidney samples. Means $\pm \mathrm{SD} .{ }^{*} \mathrm{p}<0.05,{ }^{*} \mathrm{p}<0.01$, vs. group A.

\section{Discussion}

In the present study, we first demonstrated that administration of the DHA-TG emulsion suppressed proteinuria in rats with NTS nephritis. There are several possible mechanisms of reduction of proteinuria by DHA-TG emulsion. Increased DHA levels in the PL fraction of PMNL suppressed PMNL migration by reducing leukotriene $B_{4}$ production [4]. Novel mediators, such as protectin converted from DHA [7], suppressed the inflammatory reaction in the heterologous phase due to NTS injection. Administration of the DHA-TG emulsion might ameliorate proteinuria in rats with NTS nephritis by inhibiting PMNL migration.

As shown in table 1, DHA and EPA levels increased after DHA-TG injection. This might have occurred because the DHA-TG emulsion contains 6.5\% EPA (see Methods) and the injected emulsion might have retroconverted to EPA in the kidneys and/or other organs. Changes in the fatty acid composition of plasma are shown in tables 2 and 3. Although DHA levels in the plasma TG fraction were significantly increased after DHA-TG injection, DHA 
Table 4. Total protein, total cholesterol, and creatinine levels $(\mathrm{mg} / \mathrm{dl})$ in plasma in the 4 groups

\begin{tabular}{lcccc}
\hline & \multicolumn{2}{l}{ NTS nephritis } & \multirow{2}{*}{ Group D } \\
\cline { 2 - 3 } & group A & group B & group C & \\
\hline Total protein & $5.9 \pm 0.3$ & $6.0 \pm 0.1$ & $6.2 \pm 0.1$ & $5.9 \pm 0.1$ \\
Total cholesterol & $73.3 \pm 16.7$ & $107.5 \pm 18.9^{*}$ & $103.0 \pm 10.4^{*}$ & $72.5 \pm 0.7$ \\
Creatinine & $0.24 \pm 0.01$ & $0.23 \pm 0.02$ & $0.23 \pm 0.01$ & $0.23 \pm 0.01$ \\
\hline
\end{tabular}

DHA emulsion (10 ml/kg; group A), $10 \mathrm{ml} / \mathrm{kg}$ of soybean oil emulsion (group B), and $10 \mathrm{ml} / \mathrm{kg}$ of saline (group C) were intraperitoneally administered to rats $24 \mathrm{~h}$ prior to NTS injection, and 0, 1, 2, 3, 4, and 5 days after the injection. Group D was the control group (no nephritis). All rats were sacrificed on day 6 to obtain plasma and kidney samples. Total protein, total cholesterol, and creatinine levels in plasma were analyzed enzymatically. Means \pm SD. ${ }^{*} \mathrm{p}<0.05$ vs. group A.

levels in the plasma PL and FFA fractions did not increase after injection. This might be due to the fact that the emulsion used in the present study was a TG form of DHA. Consequently, DHA levels in the plasma TG fraction alone might have increased after injecting the emulsion compared to those in the PL or FFA fraction.

In the present study, histological examination revealed no significant differences among groups A, B, and C, possibly because the NTS nephritis model might not be able to distinguish the differences in mesangial proliferative glomerulonephritis. Total plasma cholesterol levels in group A were lower than those in groups B and C (table 4). This result suggests that DHA might accelerate the metabolism of lipoproteins, such as chylomicrons or verylow-density cholesterol. Kielar et al. [8] demonstrated that DHA ameliorated murine ischemic acute renal failure. Halade et al. [9] reported that DHA attenuated kidney disease and prolonged the life span of autoimmune lupus-prone mice. However, reports on the effects of DHA-TG on proteinuria in NTS nephritis have not been published to date.

In conclusion, we demonstrated that the administration of the DHA-TG emulsion decreased proteinuria in rats with NTS nephritis in this preliminary study. These beneficial effects might be due to the suppressive effects of DHA on PMNL.

\section{Acknowledgments}

We are grateful to Shizuko Takebe for technical assistance and to Kentech (Toyama, Japan) for donating DHA.

\section{Disclosure Statement}

There is no conflict of interest.

\section{References}

1 Dyerberg J: Linoleate-derived polyunsaturated fatty acids and prevention of atherosclerosis. Nutr Rev 1986;44:125-134.

2 Calder PC: Polyunsaturated fatty acids and inflammatory processes: new twists in an old tale. Biochimie 2009;91:791-795. 
3 Masugi M: Über die experimentalle Glomerulonephritis durch das spezifische Antinierenserum. Ein Beitrag zur Pathogenese der diffusen Glomerulonephritis. Beitr Pathol 1934;92:926-966.

4 Nakamura N, Hamazaki T, Yamazaki K, et al: Intravenous infusion of tridocosahexaenoyl-glycerol emulsion into rabbits - effects on leukotriene $\mathrm{B}_{4 / 5}$ production and fatty acid composition of plasma and leukocytes. J Clin Invest 1993;92:1253-1261.

5 Geyer RP, Olsen FR, Andrus SB, et al: Preparation of fat emulsion for intravenous alimentation. J Am Oil Chem Soc 1955;32:35-370.

6 Bligh EG, Dyer WJ: Rapid method of total lipid extraction and purification. Can J Biochem Physiol 1959;37:911-917.

7 Schwab JM, Chiang N, Arita M, Serhan CN: Resolvin E1 and protectin D1 activate inflammationresolution programmes. Nature 2007;447:869-874.

8 Kielar ML, Jeyarajah DR, Zhou XJ, Lu CY: Docosahexaenoic acid ameliorates murine ischemic acute renal failure and prevents increases in mRNA abundance for both TNF- $\alpha$ and inducible nitric oxide synthase. J Am Soc Nephrol 2003;14:389-396.

9 Halade GV, Rahman MM, Bhattacharya A, et al: Docosahexaenoic acid-enriched fish oil attenuates kidney disease and prolongs median and maximal life span of autoimmune lupus-prone mice. J Immunol 2010;184:5280-5286. 